\title{
The Use of Acupuncture as an Alternative Treatment Method for Ovarian Dys- function: A Narrative Review
}

\author{
Jingyu X $\mathbf{u}^{1,2}$, Yihua Fan ${ }^{1,2}$, Junze Geng ${ }^{1,2}$, Yunzhi Zhang ${ }^{3}$, and Tian Xia ${ }^{1,2, *}$ \\ ${ }^{1}$ First Teaching Hospital of Tianjin University of Traditional Chinese Medicine, Tianjin 300193, China \\ ${ }^{2}$ National Clinical Research Center for Chinese Medicine Acupuncture and Moxibustion, Tianjin 300193, China \\ ${ }^{3}$ Tianjin University of Traditional Chinese Medicine, Tianjin 300000, China
}

*Corresponding authors: Tian Xia, First Teaching Hospital of Tianjin University of Traditional Chinese Medicine, Tianjin 300193, China, E-mail: xiatian76@163.com

Received: 08 Jul, 2021 | Accepted: 16 Jul, 2021 | Published: 26 Jul, 2021

Citation: Xu J, Fan Y, Geng J, Zhang Y, Xia T (2021) The Use of Acupuncture as an Alternative Treatment Method for Ovarian Dysfunction: A Narrative Review. J Clin Case Stu 6(3): dx.doi.org/10.16966/2471-4925.228

Copyright: (c) 2021 Xu J, et al. This is an open-access article distributed under the terms of the Creative Commons Attribution License, which permits unrestricted use, distribution, and reproduction in any medium, provided the original author and source are credited.

\begin{abstract}
It is well known that diminished ovarian reserve is one of the main causes of infertility in older women. Also, due to the delayed reproductive age of women, there is a gradual increase in the number of elderly women using assisted reproduction techniques to assist pregnancy. However, due to the decrease in ovarian function, the sensitivity of ovaries to gonadotropin $(\mathrm{Gn})$ is reduced and follicles fail to develop and mature to ovulation, which is one of the important reasons for cancelling cycles during the application of assisted reproduction for ovulation. Therefore, improving ovarian function is an important factor in improving pregnancy rates in advanced women during assisted conception. Modern medicine has no clear drugs to restore ovarian function in women, while acupuncture, as an effective and simple complementary and alternative therapy, has gradually become a hot spot for application in patients with reduced ovarian function in recent years. Therefore, this paper aims to investigate the mechanism, clinical efficacy and future application prospects of acupuncture in improving ovarian function in women.
\end{abstract}

Keywords: Acupuncture; Diminished ovarian reserve; Infertility; Complementary and alternative therapies

Abbreviations: DOR: Diminished Ovarian Reserve; HRT: Hormone Replacement Therapy; HPO: Hypothalamic-Pituitary-Ovarian; DA: Dopamine, GABA: $\gamma$-Aminobutyric Acid; $\beta$-EP: $\beta$-Endorphin; Glu: Glutamate; Asp: Aspartate; GnRH: Gonadotropin-Releasing Hormone; FSH: Follicle-Stimulating Hormone; LH: Luteinizing Hormone; E2: Estradiol; AMH: Anti-Mullerian Hormone; PI3K: Phosphatidylinositol 3-Kinase; P-Akt: Phosphorylated Akt

\section{Introduction}

With the progress of time and socioeconomic development, the role of women is gradually diversifying and female fertility problems are becoming more apparent, such as delayed fertility and reduced fertility [1]. Reproductive aging and consequent diminished ovarian reserve are becoming increasingly common causes of female infertility [2], which is mainly manifested by a decrease in the number and quality of follicles and a decrease in ovarian secretion. According to some studies, the incidence of diminished ovarian reserve (DOR) has been increasing year by year in recent years with a trend of youngeronset [3], with a clinical incidence of about 1\%-3\% [4]. Women with diminished ovarian reserve often suffer from irregular menstruation or even amenorrhea, infertility, menopausal symptoms, anxiety, and depression [5,6], which seriously affects women's health and fertility.

Hormone replacement therapy (HRT) is currently used for female diminished ovarian reserve [7] as a way to mimic the hormonal changes in normal women and to improve their quality of life and associated health risk factors. In contrast, acupuncture, as a non- pharmacological complementary and replacement therapy, has fewer adverse effects in clinical applications [8] and has gained worldwide attention for its remarkable efficacy in the field of reproduction and infertility. Many studies have shown that acupuncture plays a positive role in improving menstrual symptom changes, reproductive hormone abnormalities, and poor follicular development due to decreased ovarian function $[9,10]$. With the increasing use of acupuncture in clinical in recent years, many scholars have also conducted more indepth studies on the specific effects of acupuncture while achieving significant curative effects. As an adjunctive treatment method with a thousand-year history of application, acupuncture is gradually becoming one of the common treatment modalities used by clinicians in today's society because of its clinical simplicity, high flexibility, and remarkable efficacy. In this paper, we will discuss in detail the mechanism, clinical efficacy, safety, and application prospects of acupuncture in the treatment of diminished ovarian reserve, and explore the potential application of acupuncture for improving ovarian function to elucidate the value of acupuncture in patients with diminished ovarian reserve. 


\section{Exploration of the Mechanism of Acupuncture in the Treatment of Diminished Ovarian Reserve}

\section{Regulation of female hypothalamic-pituitary-ovarian axis (HPO) and sex hormone levels}

The female HPO axis is the basis of female reproductive endocrine homeostasis, and the balance of reproductive hormones is closely related to ovarian function. Acupuncture can stimulate peripheral nerves to produce acupuncture impulses and transmit them to the center, which can produce a series of neurohumoral regulations by regulating the activity of neurons in the hypothalamus related to the reproductive endocrine system [11] and produce regulatory effects through the female HPO axis. Zhang YM, et al. [12] found through animal experiments in rats that acupuncture could indeed effectively regulate the HPO axis in females, restoring the disturbed reproductive endocrine system to the normal dynamic balance of the body [13]. In a study by Sun YX, et al. [14], acupuncture was shown to increase the release of hypothalamic inhibitory neurotransmitters such as dopamine (DA), $\gamma$-aminobutyric acid (GABA), and $\beta$-endorphin ( $\beta$ $\mathrm{EP})$ and decrease the release of excitatory neurotransmitters such as glutamate (Glu) and aspartate (Asp) in de-ovulatory rats, resulting in a hyper-release of hypothalamic gonadotropin-releasing hormone (GnRH) This reduces the release of hypothalamic gonadotropinreleasing hormone $(\mathrm{GnRH})$, thereby reducing serum folliclestimulating hormone (FSH) levels, and in this way improves the overall reproductive hormone levels in women through feedback regulation of the HPO axis.

It is known that ovarian hypofunction will directly lead to a decrease in ovarian production of estrogen and progesterone, and a decrease in the number and/or function of receptors on the surface of follicular membrane cells, which in turn negatively regulates the female HPO axis, resulting in high production of GnRH, FSH, and luteinizing hormone $(\mathrm{LH})$. As a result, women with reduced ovarian function have lower serum estrogen and progesterone and higher FSH compared to healthy women of the same age. It has been shown that acupuncture can improve sex hormone levels by regulating the HPO axis, thereby restoring ovarian function. Cheng K, et al. [15] observed the effects of acupuncture on the HPO axis in de-ovulatory rats by acupuncture at "Guan Yuan" and "San Yin Jiao" points, and the results showed that acupuncture could effectively improve low estrogen and reduce serum hypogonadotropic hormone in rats; this suggests that acupuncture can improve abnormal reproductive hormone levels by regulating the HPO axis in de-ovulatory rats. This suggests that acupuncture can improve the abnormal reproductive hormone levels by regulating the HPO axis in de-ovulatory rats. Li Y, et al. [16] observed the effect of electro acupuncture on natural perimenopausal rats: compared with the model group, the level of estradiol (E2) in the electro acupuncture group was significantly higher, and the levels of FSH, LH, and GnRH were all decreased to different degrees. Zhang J, et al. [17] showed by systematically evaluating the efficacy of acupuncture on diminished ovarian reserve that acupuncture reduced serum FSH and LH levels more dramatically compared to HRT. This shows that acupuncture is an effective treatment modality for patients who have poor HRT results, are not suitable for HRT therapy, or prefer non-pharmacological therapies. Meanwhile, a study by Wang P, et al. [18] demonstrated that acupuncture can also activate the dopamine system in the body to improve the body's immunity and restore functional levels by affecting neuroendocrine [19], thus improving ovarian reserve function.

\section{Directly improve ovarian tissue and function}

Nrf2 is a member of the alkaline leucine zipper family of transcription factors, which regulates the transcription of a variety of antioxidant stress and anti-inflammatory genes, and its activation is one of the main defense mechanisms of cells [20], while HO-1 is one of the downstream antioxidant response elements of Nrf2. Expression of both Nrf2 and HO-1 has anti-inflammatory effects, and it reduces tissue and organ damage by regulating the inflammatory response [21,22]. In contrast, NLRP3 promotes the secretion of pro-inflammatory cytokines, exacerbates cell damage, and induces apoptosis [23]. Inhibition of NLRP3 mRNA expression reduces the inflammatory response and plays a protective role for tissues [24]. A study by Lu G, et al. [25] showed that compared with the model group of rats with ovarian atrophy, the ovaries of the group of rats treated with acupuncture after ovarian atrophy showed larger sinus follicles under light microscopy, more granulosa cell layer than the model group, better luteal development, fewer atretic follicles, and less inflammatory infiltration; it was also found that acupuncture could increase the expression of Nrf2 and HO-1 mRNA in the ovarian tissue of rats by it was also found that acupuncture could improve ovarian function by increasing the expression of Nrf2 and HO-1 mRNA and decreasing the expression of NLRP3 mRNA in rat ovarian tissue, thereby increasing ovarian anti-mullerian hormone (AMH) and E2 content and decreasing FSH through negative feedback regulation.

When the body undergoes oxidative reactions, large amounts of reactive oxygen species are produced in the body, which in turn stimulates the body to initiate the apoptotic program, when the proapoptotic factor bax in the cytoplasm is transferred to the mitochondria to combine with the anti-apoptotic member BCL-2, causing changes in both the permeability and membrane potential of the mitochondrial membrane, resulting in the release of large amounts of cytochrome $\mathrm{C}$ to induce apoptosis [26,27]. In contrast, under physiological conditions, the phosphatidylinositol 3-kinase (PI3K) signaling pathway is crucial for activating follicle growth and improving ovarian function due to its role in inducing proliferation and activation of ovarian granulosa cells. Phosphorylated Akt (P-Akt) can activate protein phosphorylation in the downstream gene family of $\mathrm{Bcl}-2$, thus enabling regulation of primordial follicle activation and development. Wang S, et al. [28] showed that acupuncture can upregulate the PI3K/Akt signaling pathway by regulating hormone (FSH, E2) levels in hypovulatory rats, resulting in increased PI3K, Akt, BCL-2 gene and protein expression levels increase, bax expression levels decrease, and P-Akt protein expression levels increase, thereby reducing granulosa cell apoptosis and activating follicle development to improve ovarian function. The study by Chen-Chen F, et al. [29] also confirmed that acupuncture could improve the number of follicles in the ovary and reduce atretic follicles; it also showed that acupuncture could reduce bax mRNA expression levels and increase BCL-2 mRNA expression levels, thus improving ovarian tissue structure and restoring ovarian function.

A study by Sun Y, et al. [14] found that the acupuncture effect could also improve the blood supply to the ovaries directly through the sympathetic nerves associated with the ovaries to the supraspinal reflex center, thus regulating the local microenvironment of the ovaries for better follicle development and maturation.

\section{Clinical Studies Related to Acupuncture in the Treat- ment of Diminished Ovarian Reserve}

Three main clinical treatments can reflect the effect of acupuncture: milli-needle, electro-acupuncture, and acupuncture point embedding. 
Age is a very important factor in the assessment of ovarian function, however, in clinical studies on the improvement of ovarian function by acupuncture, the age of the patient is a fixed value, so the assessment of ovarian function in the study is mainly in the following areas: sex hormone levels, AMH, Antral follicle count (AFC), and ovarian arterial flow index. As ovarian hypofunction ultimately affects female fertility, the clinical pregnancy rate can also be used as one of the evaluation indicators.

\section{Milli-needle acupuncture}

As the most traditional and simple method of acupuncture therapy, milli-needle acupuncture is the most widely used in clinical applications, and only a few milli-needles are used to complete the treatment. A prospective study by Shang J, et al. [30] showed that compared with the pre-needling period, FSH and LH decreased and $\mathrm{E} 2$ and $\mathrm{AMH}$ increased in patients after treatment, while AFC did not change significantly; this indicates that acupuncture is more likely to improve ovarian function by regulating reproductive endocrine levels. In the present analysis, it was found that patients with sporadic menstruation due to ovarian hypofunction showed a more significant improvement in pregnancy rate after acupuncture treatment than those with amenorrhea, suggesting that acupuncture can improve menstrual infertility due to ovarian hypofunction to a certain extent. A prospective study by Wang F, et al. [31] also confirmed that acupuncture significantly increased serum E2 levels in patients with ovarian hypofunction. Li XT, et al. [32] showed in a randomized controlled trial that the patient's blood FSH and FSH/LH levels decreased and E2 and AFC levels increased after acupuncture treatment and at follow-up compared with those before treatment; and the clinical pregnancy rate after treatment was as high as $15 \%$, suggesting that acupuncture has some effect in promoting pregnancy by improving ovarian function.

\section{Electro-acupuncture}

Electro-acupuncture is a treatment method combining millineedle and electrical stimulation to enhance the effect of milli-needle acupuncture through electrical stimulation. It can not only improve the therapeutic effect of milli-needle acupuncture but also expand the scope of milli-needle acupuncture treatment, which is now widely used in clinical. A study by Li R, et al. [33] showed that low-frequency electrical stimulation intervention (electrical frequency often $2 \mathrm{~Hz}$ ) for 1 to 3 months on top of manual cycles in patients with diminished ovarian reserve before undergoing IVF-ET could improve ovarian blood supply, reduce ovarian arterial PI and RI index, and lower the patient's blood FSH and LH levels.

\section{Embedding of acupuncture points}

Acupoint sutures are embedded in the corresponding acupuncture points, and the continuous stimulation of the buried area by the lamb's intestine sutures achieves the effect of healing and health care, and its stimulating effect in the body is similar to the long time acupuncture effect, so it is a kind of acupuncture treatment [34]. A randomized controlled trial by Zhang L, et al. [35] showed that the combination of acupoint embedding and HRT showed more significant improvements in $\mathrm{AMH}$ and $\mathrm{E} 2$ levels and reductions in serum FSH, $\mathrm{LH}$, and FSH/LH values compared with the HRT group alone. This shows that acupoint embedding as a complementary treatment is beneficial in improving the ovarian function of patients.

\section{The Safety of Acupuncture Therapy}

However, if the operator is negligent or if the needling technique is inappropriate, or if the patient is weak and nervous, adverse reactions such as dizziness, stagnant needles, hematoma, skin allergy, and infection may still occur during the treatment process; and for patients with diminished ovarian reserve, the needling points are mostly concentrated in the lower abdomen and lower back, which may cause abdominal cavity damage due to improper angle and depth of needling. Injuries to the abdominal cavity can be caused by improper angles and depths of needling but can be completely avoided if done properly. Zheng W, et al. [36] summarized and analyzed all studies that reported adverse acupuncture events and found that improper acupuncture techniques and inadequate sterilization were the main causes of adverse events. This shows that although acupuncture is simple and easy to operate, it still requires clinical practitioners to have a profound knowledge of anatomy and a good grasp of acupuncture techniques and the management of adverse events.

\section{Summary and Outlook}

As a complementary and alternative therapy for the treatment of diminished ovarian reserve, current clinical evidence suggests that acupuncture can improve patients' high serum FSH and low E2 levels and improve overall ovarian function by regulating the homeostasis of the reproductive endocrine axis in women. As a treatment that has existed for many years, acupuncture has a wide and flexible application in clinical evidence. In recent years, it has been found that acupuncture plays a better role in female reproduction and is a complementary and alternative therapy worthy of current attention and research because of its non-pharmaceutical properties that circumvent many adverse effects. The possible mechanism of action is that the acupuncture effect produces nerve conduction by stimulating the body's acupuncture points, which in turn regulates the female hypothalamic-pituitary-ovarian axis in both directions, bringing the secretion of female reproductive hormones into balance and thus achieving normal ovarian function. At the same time, the acupuncture effect is transmitted to the spinal cord center to directly improve the blood supply to the ovaries and maintain the local microenvironmental homeostasis of the ovaries.

The root cause of female ovarian hypofunction is the generation of large amounts of reactive oxygen species due to oxidative stress in the ovary, which mediates the inflammatory response and activates the apoptotic system; acupuncture can activate the transcription and translation of the anti-apoptotic member BCL-2 by upregulating the PI3K/Akt signaling pathway, stimulate the expression of antiinflammatory genes $\mathrm{Nrf} 2$ and $\mathrm{HO}-1$ in ovarian cells, thereby reducing the damage to ovarian tissue caused by inflammation and regulating follicular cell proliferation and development. This can reduce ovarian tissue damage caused by inflammation, regulate follicle cell proliferation and development, and improve ovarian function. In a basic study, it was found that the number of sinus follicles and the number of granulosa cells in the ovaries of rats with ovarian atrophy increased after acupuncture treatment.

At the same time, a large number of randomized controlled clinical trials have confirmed that acupuncture can indeed improve the problem of high FSH and low E2 in women with reduced ovarian function, thus achieving normal female physiological functions such as follicular development and regular menstruation. In addition, for women using IVF-ET, acupuncture can increase the number of eggs, quality follicles, and quality embryos, and improve the clinical pregnancy rate. This all indicates the value of acupuncture in patients with diminished ovarian reserve.

As a non-pharmacological treatment, acupuncture has fewer side effects than drugs and is quicker and easier to use than surgical 
methods. However, as a medical treatment, acupuncture requires professional training, and even if it is easy to perform, it must be done carefully. Although acupuncture is widely used in clinical practice and its efficacy is evident, the occurrence of adverse events such as stagnant needles, dizziness, hematoma, and infection still warrants clinician's vigilance. The sterilization process should be strictly controlled during the use of needling, the appropriate depth of needling should be mastered, and the patient's status should be asked before treatment is administered.

In summary, acupuncture is worthy of application in female patients with diminished ovarian reserve, which is beneficial for female health and fertility. However, due to the flexibility of acupuncture and the abundance of acupuncture points in the human body, there is still a lack of large sample and multicenter trials to investigate the most effective general combinations of acupuncture points and acupuncture treatment protocols to improve ovarian function in women. At the same time, the specific neuroendocrine mechanisms of the action of acupuncture on the human body also deserve to be explored in greater depth, which would be useful in understanding the multifaceted effects of the neurotransmission produced by acupuncture. In conclusion, acupuncture has good potential to improve ovarian function in women and is worthy of promotion, and we look forward to reporting more relevant findings in the future.

\section{Conflicts of Interest}

The authors declare that they have no conflicts of interest.

\section{References}

1. Nguyen HH, Milatn F, Vincent A (2017) Premature ovarian insufficiency in general practice: Meeting the needs of women. Aust Fam Physician 46: 360-366.

2. Kasapoğlu I, Seli E (2020) Mitochondrial Dysfunction and Ovarian Aging. Endocrinology 161: bqaa001.

3. Scott RTJr, Hofmann GE (1995) Prognostic assessment of ovarian reserve. Fertil Steril 63: 1-11.

4. Feichtinger $M$, Barnea ER, Nyachieo A, Brännström M, Kim SS (2018) Allogeneic ovarian transplantation using immunomodulator preimplantation factor (PIF) as monotherapy restored ovarian function in olive baboon. J Assist Reprod Genet 35: 81-89.

5. Cowri V, Ai-Shukri M, Ai-Farsi FA, Al-Busaidi NA, Dennison D, et al. (2015) Aetiological profile of women presenting with premature ovarian failure to a singletertiary care center in Oman. Post Reprod Health 21: 63-68.

6. Sullivan SD, Sarrel PM, Nelson LM (2016) Hormone replacement therapy in young women with primary ovarian insufficiency and early menopause. Fertil Steril 106: 1588-1599.

7. Paciuc J (2020) Hormone Therapy in Menopause. Adv Exp Med Biol 1242: 89-120.

8. Mallory MJ, Do A, Bublitz SE, Veleber SJ, Bauer BA, et al. (2016) Puncturing the myths of acupuncture. J Integr Med 14: 311-314.

9. Li X, Zhang S (2017) Professor LAI Xinsheng's clinical experience in the treatment of premature ovarian failure with tongyuan acupuncture technique and Chinese herbal medicine. Zhongguo Zhen Jiu 37: 303-306.

10. Lin J, Li XL, Song H, Li Q, Wang MY, et al. (2017) A general description for Chinese medicine in treating premature ovarian failure. Chin J Integr Med 23: 91-97.
11. Zhu H, Nan S, Suo C, Zhang Q, Hu M, et al. (2019) ElectroAcupuncture Affects the Activity of the Hypothalamic-PituitaryOvary Axis in Female Rats. Front Physiol 10: 466.

12. Zhang Y, Yu B, Chen J, Zhao Z, Jiali W, et al. (2015) Effects of acupuncture on PI3K/Akt/mTOR signaling pathway in rats with premature ovarian failure. Zhongguo Zhen Jiu 35: 53-58.

13. Wang J, Cheng K, Qin Z, Wang Y, Zhai L, et al. (2017) Effects of electroacupuncture at Guanyuan (CV 4) or Sanyinjiao (SP 6) on hypothalamus-pituitary-ovary axis and spatial learning and memory in female SAMP8 mice. J Tradit Chin Med 37: 96-100.

14. Yixuan S, Cong Q (2015) Research progress of acupuncture on improving ovarian function. Electronic J clin med literature 2: 2906.

15. Kai C, Suling T (2012) Effect of counter acupuncture "Guanyuan" and "Sanyinjiao" on hypothalamus pituitary ovary axis in ovariectomized rats. Acupunct Res 37: 15-19.

16. Li Y, Xu L, Qin Z (2014) Effects of electroacupuncture stimulation of "Sanyinjiao" (SP 6) on hypothalamus'-pituitary-ovary axis in perimenopausal rats. Acupunct Res 39: 198-201.

17. Zhang J, Huang X, Liu Y, He Y, Yu H (2020) A comparison of the effects of Chinese non-pharmaceutical therapies for premature ovarian failure: A PRISMA-compliant systematic review and network metaanalysis. Medicine (Baltimore) 99: e20958.

18. Peiwen W (2015) Try acupuncture for premature ovarian failure. Health times 13.

19. Xia L, Xia Y (2018) Clinical research and the effect mechanism on premature ovarian failure treated with acupuncture in recent 20 years. Zhongguo Zhenjiu 38: 5653-5670.

20. Steel R, Cowan J, Payerne E, O'Connell MA, Searcey M (2012) Antiinflam- matory effect of a cell-penetrating peptide targeting the Nrf2/Keap1 interaction. ACS Med Chem Lett 3: 407-410.

21. Yan J, Li J, Zhang L, Sun Y, Jiang J, et al. (2018) Nrf2 protects against acute lung injury and inflammation by modulating TLR4 and Akt signaling. Free Radic Biol Med 121: 78-85.

22. Kong X, Thimmulappa R, Craciun F, Harvey C, Singh A, et al. (2011) Enhancing Nrf2 pathway by disruption of Keap1 in mye-loid leukocytes protects against sepsis. Am J Respir Crit Care Med 184: 928-938.

23. Shao BZ, Xu ZQ, Han BZ, Su DF, Liu C (2015) NLRP3 inflammasome and its inhibitors: a review. Front Pharmacol 6: 262.

24. Kelley N, Jeltema D, Duan Y, He Y (2019) The NLRP3 Inflammasome: An Overview of Mechanisms of Activation and Regulation. Int J Mol Sci 20: 3328.

25. Ge L, Zijing X, Qian W, Qian L, Hongxiao L, et al. (2020) Effects of acupuncture on serum inflammatory factors and Nrf2/HO-1/NLRP3 signaling pathway in ovaries of rats with decreased ovarian reserve function. J tradit Chin Med 15: 1350-1356.

26. Ma M, Chen XY, Gu C, Xiao XR, Guo T, et al. (2014) Biochemical changes of oxidative stress in premature ovarian insufficiency induced by tripterygium glycosides. Int J Clin Exp Pathol 7: 88558861.

27. An J, Chen Y, Huang Z (2004) Critical upstream signals of cytochrome C release induced by a novel Bcl-2 inhibitor. J Biol Chem 279: 1913319140.

28. Wang S, Lin S, Zhu M, Li C, Chen S, et al. (2019) Acupuncture Reduces Apoptosis of Granulosa Cells in Rats with Premature Ovarian Failure via Restoring the PI3K/Akt Signaling Pathway. Int J Mol Sci 20: 6311. 
29. Fang CC, Xu QQ, Shen J, Li Q, Shen MH (2019) Effect of acupuncture on oxidative stress and apoptosis in ovarian hypofunction induced by Tripterygium Glycosides. Zhen Ci Yan Jiu 44: 810-816.

30. Jie S (2018) Prospective case series study on acupuncture treatment of premature ovarian insufficiency. Chin Acad tradit Chin Med.

31. Wang F, Fang YG, Chen YR, Bai Y, Wu X, et al. (2014) Acupuncture for premature ovarian failure: a prospective cohort study. Zhongguo Zhen Jiu 34: 653-656.

32. Li X, Xu H, Fang Y, Shang J, Yang H, et al. (2017) Acupuncture with regulating menstruation to promote pregnancy for diminished ovarian reverse: a prospective case series study. Zhongguo Zhen Jiu 37: 1061-1065.
33. Rong L, Wei S, Ge L (2016) Expert consensus on the application of electroacupuncture/transcutaneous acupoint electrical stimulation in reproductive medicine. Reprod Contracept 36: 527-535.

34. Huo J, Zhao J, Yuan Y, Wang J (2017) Research status of the effect mechanism on catgut-point embedding therapy. Zhongguo Zhen Jiu 37: 1251-1254.

35. Linyun Z, Yansheng Y, Wang L, Xiue H, Yinying L, et al. (2021) Clinical study on acupoint catgut embedding in the treatment of infertility due to ovarian dysfunction. China Health Standard Management 5: 121-124.

36. Wenke Z, Hongcai S (2011) Analysis of safety and related factors of acupuncture therapy. Chin J tradit Chin Med 12: 2777-2781. 